\title{
Metabolic engineering of Escherichia coli for production of valerenadiene
}

S. Eric Nybo ${ }^{1, \dagger}$, Jacqueline Saunders ${ }^{1}$, Sean P. McCormick ${ }^{2}$

${ }^{1}$ Ferris State University, College of Pharmacy, Department of Pharmaceutical Sciences, Big Rapids, MI

4 49307, USA

$5 \quad 2$ Ferris State University, Department of Physical Sciences, Big Rapids, MI 49307, USA

6 † Corresponding Author: EricNybo@ferris.edu

\section{Abstract}

9 Valeriana officinalis is a medicinal herb which produces a suite of compounds in its root tissue useful for 10 treatment of anxiety and insomnia. The sesquiterpene components of the root extract, valerenic acid and 11 valerena-1,10-diene, are thought to contribute to most of the observed anxiolytic of Valerian root 12 preparations. However, valerenic acid and its biosynthetic intermediates are only produced in low 13 quantities in the roots of $V$. officinalis. Thus, in this report, Escherichia coli was metabolically engineered

14 to produce substantial quantities of valerena-1,10-diene in shake flask fermentations with decane overlay. 15 Expression of the wildtype valerenadiene synthase gene (pZE-wvds) resulted in production of $12 \mu \mathrm{g} / \mathrm{mL}$ 16 in LB cultures using endogenous FPP metabolism. Expression of a codon-optimized version of the 17 valerenadiene synthase gene (pZE-cvds) resulted in 3-fold higher titers of valerenadiene $(32 \mu \mathrm{g} / \mathrm{mL})$. Co18 expression of pZE-cvds with an engineered methyl erythritol phosphate (MEP) pathway improved valerenadiene titers 65 -fold to $2.09 \mathrm{mg} / \mathrm{L}$ valerenadiene. Optimization of the fermentation medium to include glycerol supplementation enhanced yields by another 5.5 -fold ( $11.0 \mathrm{mg} / \mathrm{L}$ valerenadiene $)$. The

21 highest production of valerenadiene resulted from engineering the codon-optimized valerenadiene

22 synthase gene under strong $\mathrm{P}_{\mathrm{trc}}$ and $\mathrm{P}_{\mathrm{T} 7}$ promoters and via co-expression of an exogenous mevalonate

23 (MVA) pathway. These efforts resulted in an E. coli production strain that produced $62.0 \mathrm{mg} / \mathrm{L}$

24 valerenadiene (19.4 mg/L/OD ${ }_{600}$ specific productivity). This $E$. coli production platform will serve as the 25 foundation for the synthesis of novel valerenic acid analogues potentially useful for the treatment of 26 anxiety disorders.

27 Keywords: Valeriana, valerenic acid, metabolic engineering, sesquiterpene, Escherichia coli 
Introduction

Valeriana officinalis is a medicinal wild herb indigenous to many habitats, and the root of this plant is used as a nutraceutical preparation (Valerian) that is currently used for the treatment of anxiety and insomnia (Bent et al., 2006). The roots of $V$. officinalis produce a suite of compounds, including valepotriate alkaloids and sesquiterpenes (Bos et al., 1996). Notably, the sesquiterpene components of Valerian root extract are hypothesized to exhibit many of the beneficial anti-anxiety and anti-insomnia effects. Of the sesquiterpenes, valerenic acid is the most potent GABA-A agonist in these extracts, while valerenal, valerenol, and valerena-1,10-diene (valerenadiene) also modulate GABA-A activity to varying extents in zebrafish and mouse models (Del Valle-Mojica and Ortíz, 2012; Takemoto et al., 2014). Most importantly, the anxiolytic effect of valerian has been demonstrated in human clinical studies in recent years (Anderson et al., 2005; Barton et al., 2011).

Valerenic acid in particular has demonstrated nanomolar binding affinity for the GABA-A receptor (Benke et al., 2009). Recently, the putative binding site of valerenic acid has been determined via docking studies and site-directed mutagenesis (Luger et al., 2015). Luger et al. modeled valerenic acid in the GABA-A receptor in a distinct cleft near the $\beta 2 / 3 N 265$ transmembrane residue. The valerenic acid C12 carboxyl group is predicted to have important hydrogen-bonding interactions with residues $\beta 3$ N265 and $\beta 1$ S265. Furthermore, the $\mathrm{C}-13, \mathrm{C}-14$, and $\mathrm{C}-15$ methyl groups of the valerenane skeleton are predicted to have significant hydrophobic interactions within the pocket at residues within this binding pocket (Figure 1) (Luger et al., 2015). In summation, these observations have renewed interest in development of novel valerenic acid analogues for structure activity relationship studies.

Despite these advances, valerenic acid is produced as a minor constituent in root tissue of Valeriana officinalis (0.7-0.9\% DW) (Bos et al., 1998). The low production of valerenic acid hinders further attempts at structure activity relationship and biological activity studies. Synthetic routes toward valerenic acid derivatives are also expensive and ecologically unsuitable. Recently, Ricigliano et al. have engineered $V$. officinalis hairy roots for enhanced production of valerenic acid, which lends credence to a metabolic engineering approach for availing these molecules (Ricigliano et al., 2016). Despite this progress, the hairy root system still produces valerenic acid at very lower titers.

As an alternative approach, metabolic engineering of microbial organisms represents a green, cost-effective approach for large-scale production of sesquiterpene pharmaceuticals (Zhang et al., 2011). For example, Escherichia coli is one such model host that affords several advantages over plant-based systems due to its fast growth kinetics and capacity to produce high-value chemicals via fermentation on simple carbon sources (Lee, 1996). Furthermore, E. coli boasts considerable genetic tools, including multiple promoters and expression vectors that establish it as an ideal host for metabolic engineering.

62 Additionally, terpenes are attractive molecules for metabolic engineering, due to their use as fragrances,

63 flavors, and advanced biofuels (Peralta-Yahya et al., 2011; Sowden et al., 2005). Subsequently, these 
observations established $E$. coli as a suitable platform for engineering of valerenic acid in this present study.

Two specialized isoprenoid biosynthetic pathways exist for production of $\mathrm{C}_{5}$ isoprenyl phosphate precursors, dimethyl allyl pyrophosphate (DMAPP) and isopentenyl pyrophosphate (IPP). Many bacteria, algae, and plant chloroplasts employ the methyl erythritol phosphate pathway (MEP), which fluxes glyceraldehyde-3-phosphate and pyruvate towards IPP and DMAPP (Figure 1) (Rohmer, 1999). Fungi and non-plant eukaryotes utilize the mevalonate pathway (MVA) to convert acetyl-coenzyme A (acetylCoA) to IPP and DMAPP via eight enzymatic steps (Figure 1) (Martin et al., 2003). Subsequently, IPP and DMAPP are concatenated into progressively longer $\mathrm{C}_{10}, \mathrm{C}_{15}$, or $\mathrm{C}_{20}$ molecules by prenyltransferase enzymes. For sesquiterpene metabolism, farnesyl pyrophosphate synthase condenses 2 IPP units and 1 DMAPP unit to produce farnesyl pyrophosphate (FPP). Subsequently, FPP can be cyclized by a variety of sesquiterpene hydrocarbons by cognate terpene synthases, such as valerenadiene synthase (VDS) (Figure 1).

However, E. coli generates only a finite pool of FPP, and while introduction of a sesquiterpene synthase results in detectable production of sesquiterpenes (Martin et al., 2001), overproduction of the molecule requires redirecting substantial carbon flux to the limited substrate FPP. For example, amorphadiene is a sesquiterpene intermediate in the biosynthesis of the antimalarial drug artemisinin. Martin and co-workers discovered that heterologous expression of the mevalonate isoprenoid pathway from yeast in E. coli leads to unregulated carbon flux towards FPP and amorphadiene, which can be semi-synthetically converted to artemisinin (Martin et al., 2003). In using this substrate-engineering approach, Keasling and co-workers have produced amorphadiene in $E$. coli at yields of $500 \mathrm{mg} \mathrm{L}^{-1}$ in shake flask (Redding-Johanson et al., 2011) and $27 \mathrm{~g} \mathrm{~L}^{-1}$ in bioreactors (Tsuruta et al., 2009). This generalized approach lends itself to the microbial synthesis of other sesquiterpenes via introduction of variant terpene synthases.

In this report, a metabolic engineering platform was developed for synthesis of valerenadiene in $E$. coli in three steps. Because plant terpene synthase genes are poorly expressed in E. coli, owing to different codon usage, first an E. coli codon-optimized version of the valerenadiene synthase gene (cvds) was synthesized. This lead to a three-fold higher production of valerenadiene over expression of the wildtype plant gene ( $w v d s$ ). Secondly, we enhanced carbon flux to FPP by designing a construct that overexpressed several rate-limiting steps of the native methyl erythritol phosphate (MEP) pathway. Thirdly, we cloned cvds under the control of $\mathrm{P}_{\mathrm{trc}}$ and $\mathrm{P}_{\mathrm{T} 7}$ promoters to further enhance terpene synthase protein levels and we co-expressed these constructs with a heterologous mevalonate pathway from baker's yeast. These efforts resulted in a high-level production strain that synthesized $62.0 \mathrm{mg} \mathrm{L}^{-1}$ valerenadiene (19.4 mg/L/OD 600 specific productivity) in shake flask cultures.

\section{Methods and Materials}


Bacterial strains and growth conditions

100

101

102

103

104

105

106

107

108

109

110

111

112

113

114

115

116

117

118

119

120

121

122

123

124

125

126

127

128

129

130

131

132

133

E. coli JM109 (New England Biolabs) was used as the host for all routine cloning manipulations, and E. coli DH5aZ1 (Expressys, Germany) and E. coli BL21(DE3) (ThermoFisher) were used as hosts for sesquiterpene production (Supplementary Table 1). E. coli DH5aZ1 overexpresses a copy of the lacla repressor on the chromosome for efficient repression of the lac operator (Lutz and Bujard, 1997). Chemically competent E. coli were generated with the E. coli Mix and Go Transformation Kit (ZYMO Research) and were transformed using standard molecular methodologies (Sambrook and W Russell, 2001). E. coli strains were grown in LB agar or LB broth at $37 \square \mathrm{C}$ for routine maintenance. For production of sesquiterpenes, E. coli DH5aZ1 derivatives were grown in 2xYT with supplemented glycerol at $30 \square \mathrm{C}$. for production of valerenadiene. For expression of the mevalonic acid pathway, bacterial growth media was buffered with phosphate buffered saline using a stock solution of 10x PBS (Sambrook and W Russell, 2001). Strains were supplemented with ampicillin $\left(100 \mu \mathrm{g} \mathrm{m}^{-1}\right)$, chloramphenicol (35 $\mu \mathrm{g}$ $\left.\mathrm{mL}^{-1}\right)$, and kanamycin $\left(50 \mu \mathrm{g} \mathrm{mL}^{-1}\right)$ as necessary. When multiple plasmids were co-expressed, the chloramphenicol and kanamycin concentrations were adjusted to one-half these amounts.

\section{Cloning of vds and engineered MEP pathway constructs}

Oligonucleotide primers were synthesized by IDT-DNA (Supplementary Table 2), and sequences were verified by sequencing analysis (ACGT, Inc.). Polymerase chain reaction was carried out using Primestar ${ }^{\circledR}$ HS Polymerase (Takara Bio.) by following the manufacturer's protocols. The wildtype valerenadiene synthase gene ( $w v d s)$ gene was amplified via polymerase chain reaction from the pET28a(+)-VoTPS1 (hereafter referred to as pET-wvds) construct as described previously (Yeo et al., 2013). The cvds gene was codon-optimized for expression in E. coli, synthesized, and spliced into cloning vector pUC57-cvds (GenScript). The cvds gene was amplified via polymerase chain reaction, and both the wvds and cvds genes were cut and spliced into the EcoRI/BamHI restriction sites of pZE12MCS under the control of the intermediate strength $\mathrm{P}_{\mathrm{LlacO} 1}$ promoter (Expressys, Germany) to afford constructs pZE-wVDS and pZE-cVDS, respectively (Lutz and Bujard, 1997). For insertion under the stronger $P_{\text {trc }}$ promoter, cvds was PCR amplified, cut, and spliced into the BamHI/EcoRI sites of pTrcHisA (Thermo Fisher Scientific) to afford pTrcHis-cvds. In this construct, $c v d s$ is fused to the $N$-terminal hexahistidine and Xpress $^{\mathrm{TM}}$ epitope tags. For comparisons involving the $\mathrm{P}_{\mathrm{T} 7}$ promoter, the $c v d s$ gene was cloned into the $\mathrm{Ncol}$ and $\mathrm{EcoRI}$ sites of $\mathrm{pET} 28 \mathrm{a}(+)$ to afford $\mathrm{pET}$-cvds. $\mathrm{pET}$-wvds features $w v d s$ fused to the $\mathrm{P}_{\mathrm{T} 7}$ promoter and was included in experiments to reflect baseline production of valerenadiene from T7polymerase driven expression of the wildtype gene.

The $d x s$-idi-fps genes were synthesized as a polycistron as previously described in vector pUC57-operon 3 (Genscript) (Bell et al., n.d.). The $d x s$-idi-fps genes were digested with EcoRI/BamHI and were spliced into the EcoRI/BamHI sites of pSTV28 to afford pSTV-dxs-idi-fps. 

plasmid \# 35151). For co-expression of valerenadiene synthase and the mevalonate pathway, E. coli competent cells were co-transformed with the relevant constructs and plated on LB agar supplemented with antibiotics. For terminal endpoint assays, E. coli strains were grown in $1 \mathrm{~mL}$ of LB supplemented with antibiotics for 12-16 hours until the strains were in stationary phase growth, and then they were inoculated into $5 \mathrm{~mL} 2 x$ YT medium containing $3 \%$ glycerol with an overlay of $1 \mathrm{~mL}$ of decane. For time course assays, E. coli strains were grown in $50 \mathrm{~mL} 2 x$ YT medium containing $3 \%$ glycerol with an overlay of $10 \mathrm{~mL}$ of decane. Time points were collected at 12, 24, 36, 48, 72, and 96 hours of 100 microliters of culture broth (for $\mathrm{OD}_{600}$ measurements) and 100 microliters of decane overlay for valerenadiene determination.

GC-MS analysis of valerenadiene

Valerenadiene production by the various strains was measured by GC-MS via selective ion monitoring for the molecular ion (204 m/z), the $189 \mathrm{~m} / \mathrm{z}$ ion, and the $69 \mathrm{~m} / \mathrm{z}$ ion, as described previously

147 (Martin et al., 2003). Cells were grown in LB or 2x YT medium with 3\% glycerol and induced with 200

148 micromolar IPTG to express the wvds or $c v d s$ gene and either the engineered MEP operon or the mevalonate pathways. Cultures were covered with a $20 \%(\mathrm{v} / \mathrm{v})$ decane overlay to trap volatile sesquiterpenes. 100 microliters of decane overlay was diluted in 900 microliters of hexane, and 1 microliter of sample was subjected to GC-MS analysis. Samples were analyzed at the Ferris State

152 University Shimadzu Core Laboratory for Academic Research Excellence (FSU-SCLARE) on a Shimadzu

153 QP-2010 Ultra GC mass spectrometer. Valerenadiene in experimental samples was compared to

154 authentic valerenadiene standard. Valerenadiene concentration was converted to beta caryophyllene 155 equivalents using a caryophyllene standard curve and the relative abundance of $69 \mathrm{~m} / \mathrm{z}, 189 \mathrm{~m} / \mathrm{z}$, and $156204 \mathrm{~m} / \mathrm{z}$ mass fragments. Valerenadiene standard was isolated as described previously (Yeo et al., 157 2013).

\section{Results}

Valerenadiene production in E. coli and gene optimization

To evaluate the production of valerenadiene synthase in E. coli, the gene encoding wildtype valerenadiene synthase ( $w v d s$ ) from $V$. officinalis was cloned under the control of the IPTG-inducible

$162 \mathrm{P}_{\text {LlacO1 }}$ promoter of high copy number expression plasmid pZE12MCS to afford pZE-wVDS. Previously, 163 other groups have reported that sesquiterpene production in E. coli is limited by poor expression of 164 cognate plant terpene synthases (Martin et al., 2001; Wang et al., 2011). We surmised that the wvds 165 gene may encode several rare codons that inhibit optimal translation in E. coli. Therefore, we 166 synthesized a codon-optimized version of the valerenadiene synthase gene for expression in $E$. coli 167 (GenScript) and fused it to the PLlacO1 to afford pZE-cVDS. pZE-wVDS, pZE-cVDS, and empty 168 pZE12MCS vector were transformed in E. coli. The E. coli lines were grown in $5 \mathrm{~mL}$ of LB medium 
overlaid with decane for 48 hours and induced with IPTG. A major valerenadiene peak was detected in extracts of pZE-wVDS and pZE-cVDS cultures that eluted the same retention time and exhibited identical mass fragmentation pattern to valerenadiene standard (Supplementary Figure 1). This peak was not detected in PZE12MCS control strains, which confirmed low production of valerenadiene in the VDSengineered lines. E. coli pZE-wVDS produced $8 \mu \mathrm{g} / \mathrm{L}$ and $12 \mu \mathrm{g} / \mathrm{L}$ valerenadiene at 24 and 48 hours, respectively, whereas $E$. coli pZE-cVDS produced 16 and $32 \mu \mathrm{g} / \mathrm{L}$ valerenadiene at 24 and 48 hours, respectively (Figure 2). This results suggests that the codon-optimized valerenadiene synthase is more efficiently expressed than the wildtype version, which results in a 3-fold improvement in valerenadiene titer.

However, the yields of valerenadiene achieved were quite low, and we surmised this was likely due to low levels of prenyl phosphate precursors (IPP, DMAPP, and FPP). The endogenous MEP pathway of E. coli produces a small pool of FPP that is the precursor for trans-octaprenyl diphosphate (ODP) in production of ubiquinone and cis, trans-undecaprenyl diphosphate (UDP) for production of peptidoglycan (Asai et al 1994, Bouhss et al 2008, Okada et al 1997). However, the production of FPP by the endogenous pathway is limited due to regulation of several key steps (Estévez et al., 2001). Therefore, we designed a construct that would overexpress rate-limiting steps of the MEP pathway to augment greater carbon flux from primary metabolism to FPP. In our previous work, we engineered Rhodobacter capsulatus to produce high levels of the triterpene botryococcene, which is biosynthesized from two molecules of FPP via squalene synthase-like enzymes (SSL-1 and SSL-3), via expression of the deoxy-xylulose phosphate synthase $(d x s)$, isopentenyl diphosphate isomerase (idl) genes from $E$. coli, and farnesyl pyrophosphate synthase (fps) gene from Gallus gallus (Khan et al., 2015). Fusion of the $d x s$-idi-fps genes to the SSL-1+3 chimeric botryococcene synthase and expression of the resulting construct generated $5 \mathrm{mg} / \mathrm{gDW}$ production of botryococcene in the $R$. capsulatus host. In a similar fashion, we fused the $d x s$-idi-fps genes under the control of the IPTG-inducible $\mathrm{P}_{\text {lacuv5 }}$ promoter of pSTV28 to generate construct pSTV-dxs-idi-fps. This construct was co-transformed with pZE-wvds and pZE-cvds, and the lines were grown in $5 \mathrm{~mL}$ LB medium with decane overlay at $30^{\circ} \mathrm{C}$ for 48 hours and induced with IPTG. Co-expression of pSTV-dxs-idi-fps with pZE-wVDS and pZE-cVDS resulted in a 14fold increase $(0.17 \mathrm{mg} / \mathrm{L})$ and a 65 -fold increase $(2.09 \mathrm{mg} / \mathrm{L})$ in production of valerenadiene, respectively (Figure 2). These results demonstrate that expression of the engineered MEP pathway greatly augmented carbon flux to FPP and valerenadiene.

\section{Effect of glycerol supplementation on valerenadiene production}

With the engineered MEP pathway construct in hand, we hypothesized that optimization of the production medium could further enhance sesquiterpene yield. In fact, Zhang et al. reported that glycerol supplementation of culture medium increased sabinene production to $>40 \mathrm{mg} / \mathrm{L}$ in $E$. coli engineered with sabinene synthase and a heterologous mevalonate pathway (Zhang et al., 2014). Furthermore, Morrone 
and co-workers engineered higher levels of $\mathrm{C}_{20}$ abietadiene production and increased biomass when glycerol supplemented media was used (e.g. increasing abietadiene specific productivity from 1 $\mathrm{mg} / \mathrm{L} / \mathrm{OD}_{600}$ without glycerol supplementation to $2.5 \mathrm{mg} / \mathrm{L} / \mathrm{OD}_{600}$ with glycerol supplementation) (Morrone et al., 2010). E. coli pZE-wVDS/pSTV-dxs-idi-fps and E. coli pZE-cVDS/pSTV-dxs-idi-fps were grown in 5 $\mathrm{mL}$ of $2 x$ YT medium supplemented with 0 to $5 \%$ glycerol. The E. colipZE-wVDS/pSTV-dxs-idi-fps valerenadiene production increased 15 -fold from $0.11 \mathrm{mg} / \mathrm{L}$ to $1.5 \mathrm{mg} / \mathrm{L}$ when supplemented with glycerol, however, further production was possibly stymied by poor heterologous expression of valerenadiene synthase. In contrast, E. coli pZE-cVDS/pSTV-dxs-idi-fps demonstrated better responsiveness to the glycerol supplementation, as valerenadiene productivity increased from $5.1 \mathrm{mg} / \mathrm{L}$ to $11.0 \mathrm{mg} / \mathrm{L}$ when supplemented with $3 \%$ glycerol (Figure 3). Therefore, $3 \%$ glycerol was used for all further experiments.

To observe production of valerenadiene over time, we conducted a time course of $E$. coli pZEwVDS/pSTV- $d x s$-idi-fps and E. coli pZE-cVDS/pSTV- $d x s$-idi-fps in 2x YT+3\% glycerol media over 72 hours. The results of the time course replicated the observations in the glycerol supplementation experiment. E. colico-expressing the engineered MEP pathway and the $w v d s$ gene reached maximal growth $\left(\mathrm{OD}_{600}=9.0\right)$ and valerenadiene production $(3 \mathrm{mg} / \mathrm{L})$ at 24 hours (Figure 4). The E. coli line coexpressing the engineered MEP pathway and $c v d s$ gene reached also reached both its greatest level of growth $\left(\mathrm{OD}_{600}=9.0\right)$ and valerenadiene production $(10.6 \mathrm{mg} / \mathrm{L})$ at 24 hours. Valerenadiene concentration decreased in both lines from 48 to 72 hours, likely due to volatilization of the sesquiterpene.

\section{Engineering of vds under the $P_{\text {trc }}$ promoter}

We hypothesized that low-level expression of the terpene synthase from the $\mathrm{P}_{\text {LlacO1 }}$ promoter might be a metabolic rate-limiting factor in the turnover of FPP substrate. Based on the previous experiment, expression of the cvds gene resulted in 5-10-fold higher quantities of valerenadiene than expression of wvds, depending on glycerol supplementation, which was inferred to be the direct result of higher concentration of terpene synthase catalyst in the E. coli cell. Therefore, the $c v d s$ gene was fused to the strong $\mathrm{P}_{\text {trc }}$ promoter to afford construct pTrcHis-cvds. This construct was expressed alone and coexpressed with pSTV-dxs-idi-fps in E. coli DH5aZ1 cells in triplicate $5 \mathrm{~mL} 2 \mathrm{xYT}+3 \%$ glycerol fermentations for 48 hours. The strain harboring pTrcHis- $c v d s$ produced $0.046 \mathrm{mg} / \mathrm{L}$ valerenadiene, whereas the strain harboring pTrcHis-cvds and pSTV- $d x s$-idi-fps produced approximately $20.3 \mathrm{mg} / \mathrm{L}$ (Supplementary Figure 2). This represents a 440-fold improvement over the pTrcHis-cvds only expression line. Furthermore, expression of the $c v d s$ gene by the $\mathrm{P}_{\text {trc }}$ promoter (e.g. by strain E. coli $\mathrm{DH} 5 \mathrm{aZ1} /(\mathrm{pTrcHis}-\mathrm{cvds}) /(\mathrm{pSTV}-\mathrm{dxs}$-idi-fps)) represented a $40 \%$ increase in valerenadiene titers as compared to expression from $\mathrm{P}_{\mathrm{LlacO} 1}$ (e.g. by strain E. coli DHaZ1/(pZE-cvds)/(pSTV-dxs-idi-fps)).

Recently, the MEP pathway has been demonstrated to be subject to endogenous regulation by $E$. coli, possibly due to feedback inhibition of the metabolite methylerythritol cyclodiphosphate (MEcDP) (Banerjee and Sharkey, 2014). We rationalized that FPP enhancement might be limited via the MEP 
pathway, therefore we co-expressed an exogenous MVA pathway to greatly augment FPP precursor levels along with $\mathrm{pTrcHis-cvds.} \mathrm{In} \mathrm{a} \mathrm{previous} \mathrm{study,} \mathrm{Peralta-Yahya} \mathrm{and} \mathrm{co-workers} \mathrm{engineered} \mathrm{a}$ heterologous MVA pathway for production of the sesquiterpene biofuel bisabolene (Peralta-Yahya et al., 2011). Expression of the construct pBbA5c-MevT(CO)-MBIS(CO, ispA) along with the codon-optimized AgBis gene resulted in production of $586 \pm 65 \mathrm{mg} / \mathrm{L}$ bisabolene. pBbA5c-MevT(CO)-MBIS(CO, ispA) features a codon-optimized, eight gene MVA pathway driven by the $\mathrm{P}_{\text {trc }}$ promoter on a medium copy number plasmid (Supplementary Table 1). The three upstream gene products (atoB, hmgs, thmgr) convert acetyl-CoA to mevalonic acid, and the five downstream gene products (erg12, erg8, mvd1, idi, ispA) convert mevalonic acid to farnesyl pyrophosphate (Figure 1). Similarly, E. coli DH5 $\alpha Z 1$ was transformed with pTrcHis-cvds and pBbA5c-MevT(CO)-MBIS(CO, ispA) and grown in triplicate $5 \mathrm{~mL}$ $2 \mathrm{xYT}+3 \%$ glycerol fermentations for 48 hours. This strain produced $42.5 \mathrm{mg} / \mathrm{L}$ valerenadiene, a 1000 -fold increase over the cvds-expressing line and a 2-fold increase over the engineered MEP pathway (Supplementary Figure 2). This result demonstrated that the mevalonic acid pathway efficiently coupled the microbial synthesis of FPP to valerenadiene synthase to produce significant levels of valerenadiene. Thus, the mevalonic acid pathway was used for further experiments. These results were further confirmed in a time course experiment of the same strain, which achieved a titer of $35 \mathrm{mg} / \mathrm{L}$ valerenadiene at 24

255 hours (Figure 5).

Co-expression of vds with the T7 RNA polymerase promoter and MVA pathway

We observed that the $\mathrm{P}_{\text {trc }}-c v d s$ gene constructs demonstrated more robust production of

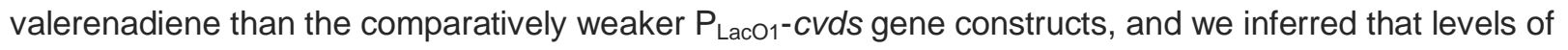

259 VDS catalyst may be rate-limiting in this latter system. Therefore, we decided to compare the expression

260 of the wvds and cvds genes from a "weak" promoter system $\left(\mathrm{P}_{\mathrm{LacO} 1}\right)$, an "intermediate/strong" promoter

261 system $\left(\mathrm{P}_{\mathrm{trc}}\right)$, and a strong promoter system $\left(\mathrm{P}_{\mathrm{T7}}\right)$ along with the mevalonic acid pathway. We

262 hypothesized that the $\mathrm{P}_{\mathrm{LacO} 1}$ promoter might not be able to drive sufficient expression of VDS to efficiently

263 turnover the abundant FPP substrate synthesized via the mevalonic acid pathway. E. coli DH5oZ1 pZE-

$264 w v d s$ and E. coli DH5 $\alpha Z 1$ pZE-cvds were grown in triplicate cultures as before and produced minimal

265 amounts of valerenadiene (Supplementary Figure 3. When pBbA5c-MevT(CO)-MBIS(CO, ispA) was co-

266 expressed in these lines, they produced $2.8 \mathrm{mg} / \mathrm{L}\left(0.497 \mathrm{mg} / \mathrm{L} / \mathrm{OD}_{600}\right)$ and $7.3 \mathrm{mg} / \mathrm{L}\left(1.03 \mathrm{mg} / \mathrm{L} / \mathrm{OD}_{600}\right)$,

267 respectively, which strongly suggested that the amount of VDS catalyst was limited (Supplementary

268 Figure 3). Expression of $c v d s$ from the $P_{\text {trc }}$ promoter with the mevalonic acid pathway increased

269 production 6-fold to $42.5 \mathrm{mg} / \mathrm{L}\left(6.07 \mathrm{mg} / \mathrm{L} / \mathrm{OD}_{600}\right)$ (Supplementary Figure 3).

Subsequently, the wvds and cvds genes were cloned into pET28a for expression under the strong 271 T7 RNA polymerase promoter. The resulting constructs, $\mathrm{pET}$-wvds and $\mathrm{pET}$-cvds were introduced into $E$. 272 coli BL21(DE3) cells to utilize the IPTG-inducible T7 RNA polymerase system. These plasmids were 273 transformed alone or in combination with the mevalonic acid pathway and grown in triplicate $2 x Y T+3 \%$ 
274 glycerol cultures for 48 hours. The pET-wvds-only line produced $0.345 \mathrm{mg} / \mathrm{L}$ valerenadiene, whereas the

275 pET-cvds-only line produced $1.09 \mathrm{mg} / \mathrm{L}$ valerenadiene (Figure 6). Co-expression of pET-wvds and

$276 \mathrm{pBbA5c-MevT(CO)-MBIS(CO}$, ispA) resulted in production of $10.5 \mathrm{mg} / \mathrm{L}$ valerenadiene $\left(3.27 \mathrm{mg} / \mathrm{L} / \mathrm{OD}_{600}\right)$

277 (Figure 7). Most importantly, when pET-cvds was introduced with the mevalonic acid pathway, the

278 highest-yielding valerenadiene strain was achieved with production of $62.0 \mathrm{mg} / \mathrm{L}$ valerenadiene (19.4

$279 \mathrm{mg} / \mathrm{L} / \mathrm{OD}_{600}$ specific productivity). These experiments demonstrated that the amount of VDS catalyst and

280 FPP substrate in vivo are both limiting factors for production of valerenadiene, which can be remedied via

281 expression of $c v d s$ from a strong $\mathrm{P}_{\mathrm{trc}} / \mathrm{P}_{\mathrm{T} 7}$ promoter and heterologous expression of the mevalonic acid

282 pathway.

\section{Discussion}

Valerenic acid is the most potent sesquiterpenoid component produced in the roots of Valeriana officinalis that exhibits GABA-A activity. Development of this compound as a novel anxiolytic drug is

286 hindered by the low production of valerenic acid by the native plant. As a first step towards the development of a production platform for valerenic acid analogues, we have employed $E$. coli as a workhorse metabolic host for engineering of terpene chemicals. In this study, valerenadiene was produced by introducing a codon-optimized valerenadiene synthase gene into Escherichia coli along with an engineered MEP pathway or Saccharomyces cerevisiae-based mevalonic acid pathway. The codonoptimized valerenadiene synthase gene was found to be a critical component for engineering high valerenadiene titers, as it resulted in 3-fold higher production of valerenadiene as compared to the wildtype sequence. This very likely indicates that translation of the codon-optimized form of the gene is very efficient. Further improvements in productivity were realized by the overexpression of the $d x s$, idi, and fps genes to augment carbon flux through the MEP pathway. This strategy resulted in a 65 -fold increase in production of valerenadiene $(2.09 \mathrm{mg} / \mathrm{L})$. This was improved a further 6 -fold by optimizing the fermentation medium to 2 XYT with $3 \%$ glycerol supplementation $(11.0 \mathrm{mg} / \mathrm{L})$. Next, the $c v d s$ gene was fused to the stronger $P_{\text {trc }}$ promoter, which resulted in approximately $40 \%$ increase in production of valerenadiene when pTrcHis-cvds was co-expressed with the engineered MEP pathway $(20 \mathrm{mg} / \mathrm{L})$. This result demonstrated the importance of driving expression of valerenadiene synthase from strong promoters to ensure ample availability of terpene synthase catalyst.

Notably, the valerenadiene synthase exhibits similar kinetic properties (e.g. $\mathrm{K}_{\mathrm{m}}$ for $\mathrm{FPP}=7.2 \mu \mathrm{M}$, $k_{\text {cat }}=5.7 \times 10^{-3} \mathrm{~s}^{-1}$ ) to other published terpene synthases (Yeo et al., 2013). For example, the wellcharacterized amorphadiene synthase exhibits a two-fold lower $\mathrm{K}_{\mathrm{m}}$ for FPP (i.e. $\mathrm{K}_{\mathrm{m}}=3.3 \mu \mathrm{M}$ and $k_{\text {cat }}=$ $6.8 \times 10^{-3} \mathrm{~s}^{-1}$ ) (Picaud et al., 2005). Further efforts to improve the catalytic functionality of valerenadiene

306 synthase could incorporate rationale modifications to the enzyme. Alternatively, further improvements in 307 terpene synthase activity have been realized via expression of a terpene synthase as a gene fusion with 308 farnesyl pyrophosphate synthase to achieve substrate channeling of FPP to the active site of VDS. Wang 
and co-workers reported that expression of ispA and apple farnesene synthase (aFS) from the same construct with the mevalonic acid pathway resulted in production of $57.6 \mathrm{mg} / \mathrm{L}$ of $\alpha$-farnesene in $E$. coli

311 (Wang et al., 2011). Fusing of ispA-aFS as a single gene construct with a (GGGGS) ${ }_{2}$ amino acid linker

312 increased production to $87.8 \mathrm{mg} / \mathrm{L}$ of $\alpha$-farnesene. Additionally, Niehaus et al. achieved substrate

313 channeling by fusing the triterpene synthases SSL-1 and SSL-3 genes to form a single gene fusion, SSL-

$3141+$ SSL-3, which increased production from $20 \mathrm{mg} / \mathrm{L}$ botryococcene to $50 \mathrm{mg} / \mathrm{L}$ botryococcene in yeast

315 (Niehaus et al., 2011).

However, the greatest increases in valerenadiene yield involved heterologous expression of the

317 Saccharomyces cerevisiae mevalonic acid pathway. Expression of the MVA pathway resulted in an

318 unregulated metabolic flux towards FPP, which could be efficiently coupled to valerenadiene synthase for

319 production of $62.0 \mathrm{mg} / \mathrm{L}$ valerenadiene in culture tubes with a specific productivity of $19.4 \mathrm{mg} / \mathrm{L} / \mathrm{OD}_{600}$.

320 This study showed that $E$. coli is capable of high-level production of valerenadiene with relatively minimal

321 optimization of the production media and commercially available expression vectors, such as pBBa5c-

322 MevT-MBIS(CO,IspA). Further efforts to improve valerenadiene yield will require optimization of the

323 upstream and downstream genetic components of the MVA pathway and subsequent scale-up

324 fermentation in bioreactors. In addition, we are conducting rational attempts to engineer CYP450s to

325 oxidize and functionalize the valerenadiene skeleton to generate a library of potential GABA-A ligands.

\section{Acknowledgements}

327 The authors gratefully acknowledge Scott Kinison, Garrett Zinck, and Dr. Joseph Chappell (University of 328 Kentucky) for the pET-wvds and cloning of the pET-cvds constructs, valerenadiene standard, and helpful 329 discussions concerning GC-MS protocols.

\section{Funding}

331 S.E.N. was supported by a NARSAD Young Investigator Grant from the Brain \& Behavior Research 332 Foundation and a Faculty Research Grant from Ferris State University. J.S. was supported by a Summer 333 Research Fellowship from Ferris State University. S.P.M. was supported by a Faculty Research Grant 334 and Ferris Merit Foundation Grant from Ferris State University. 
Figure 1 Biosynthesis of valerenadiene via the methyl erythritol phosphate pathway (MEP, pathway A) and the mevalonic acid pathway (MVA, pathway B). IPP and DMAPP are synthesized via the MEP or MVA pathways via different upstream routes. The MEP pathway is initiated via condensation of glyceraldehyde 3-phosphate and pyruvate from glycolysis via 1-deoxy-D-xylulose 5-phosphate synthase (DXS), and the MVA pathway is initiated via condensation of two molecules of acetyl-CoA. Two molecules of IPP and one molecule of DMAPP are condensed into $C_{15}$ farnesyl pyrophosphate (FPP). FPP is cyclized into valerenadiene via valerenadiene synthase (VDS). The abbreviations of the enzymes are as follows: DXS, 1-deoxy-D-xylulose 5-phosphate synthase; atoB, thiolase; HMGS, hydroxymethylglutaryl-CoA synthase; HMGR, truncated hydroxy-methylglutaryl-CoA reductase; ERG12, mevalonate kinase; ERG8, phosphomevalonate kinase; MVD1, diphosphomevalonate decarboxylase; IDI, isopentenyl diphosphate isomerase; FPS, farnesyl pyrophosphate synthase from Gallus gallus; IspA, farnesyl pyrophosphate synthase from Escherichia coli; VDS, valerenadiene synthase.

Figure 2 Engineering of wildtype (pZE-wvds) and codon-optimized (pZE-cvds) valerenadiene synthase. (A) E. coli engineered with empty vector control, wildtype, and codon optimized VDS genes was grown for 24 (white bars) and 48 hours (black bars) and yield of valerenadiene was determined. (B) Growth of engineered $E$. coli lines was determined by measuring optical density $\left(\mathrm{OD}_{600}\right)$ at 24 (white bars) and 48 hours (black bars), respectively. (C) E. coli strains were co-transformed with empty vector control, wildtype, or codon-optimized $v d s$ and pSTV-dxs-idi-fps for enhancement of the endogenous MEP pathway. (D) Growth of engineered E. coli lines was determined by measuring optical density $\left(\mathrm{OD}_{600}\right)$. Strains were inoculated in $5 \mathrm{~mL}$ cultures of LB media with $1 \mathrm{~mL}$ decane overlay and experiments were carried out in triplicate. Error bars indicate standard error of the mean.

Figure 3 Effect of glycerol conditions on production of valerenadiene. E. coli strains engineered with pZE- $w v d s$ and pSTV-dxs-idi-fps or pZE-cvds and pSTV-dxs-idi-fps were grown in $5 \mathrm{~mL} 2 x Y T$ media with $0 \%-4 \%$ glycerol and $1 \mathrm{~mL}$ decane overlay for 48 hours and valerenadiene yield was determined. Experiments were carried out in triplicate and error bars indicate standard error of the mean.

Figure 4 Time course of $E$. coli expressing an engineered MEP pathway and valerenadiene synthase. E. coli DH5aZ1 strains harboring the engineered MEP construct pSTV- $d x s$-idi-fps were co-expressed with the wildtype (pZE-wvds, solid line) or codon-optimized (pZE-cvds, dotted line) valerenadiene synthase gene. Cultures were grown in $50 \mathrm{~mL} 2 x \mathrm{YT}+3 \%$ glycerol with $10 \mathrm{~mL}$ decane overlay for 72 hours. (Left panel) Valerenadiene yield $\left(\mathrm{mg} \mathrm{L}^{-1}\right)$ and (right panel) biomass $\left(\mathrm{OD}_{600}\right)$ were determined from double triplicate experiments. Error bars indicate the standard error of the mean.

Figure 5 Time course experiment of E. coli co-expressing pTrcHis-cvds and the Saccharomyces cerevisiae MVA pathway. E. coli DH5aZ1 strains harboring the $\mathrm{pBBa} 5 \mathrm{c}-\mathrm{MevT}+\mathrm{MBIS}(\mathrm{CO}, \mathrm{IspA})$ and pTrcHis-cvds constructs were grown in $50 \mathrm{~mL} 2 x \mathrm{YT}+3 \%$ glycerol with $10 \mathrm{~mL}$ decane overlay for 72 hours. (Left panel) Valerenadiene yield $\left(\mathrm{mg} \mathrm{L}^{-1}\right)$ and (right panel) biomass $\left(\mathrm{OD}_{600}\right)$ were determined from double triplicate experiments. Error bars indicate the standard error of the mean.

Figure 6 Production of valerenadiene using the T7 RNA polymerase to drive expression of $v d s$. E. coli BL21(DE3) strains harboring empty vector control, pET-wvds, and pET-cvds were expressed with and without the Saccharomyces cerevisiae MVA pathway (pBBa5c-MevT-MBIS(CO, IspA)). Strains were grown in triplicate $5 \mathrm{~mL} 2 x Y T$ media $+3 \%$ glycerol fermentations with $1 \mathrm{~mL}$ decane overlay for 48 hours. (Left panel) valerenadiene volumetric production (mg/L) was determined after 48 hours. (Right panel) Valerenadiene production was normalized by dividing volumetric production by the biomass after 48 hours $\left(\mathrm{mg} / \mathrm{L} / \mathrm{OD}_{600}\right)$. 
bioRxiv preprint doi: https://doi.org/10.1101/148338; this version posted June 9, 2017. The copyright holder for this preprint (which was not certified by peer review) is the author/funder. All rights reserved. No reuse allowed without permission.

384

385

386

387

388 


\section{References}

Anderson, G.D., Elmer, G.W., Kantor, E.D., Templeton, I.E., Vitiello, M. V., 2005. Pharmacokinetics of valerenic acid after administration of valerian in healthy subjects. Phyther. Res. 19, 801-803. doi:10.1002/ptr.1742

Banerjee, a, Sharkey, T.D., 2014. Methylerythritol 4-phosphate (MEP) pathway metabolic regulation. Nat. Prod. Rep. 31, 1043-55. doi:10.1039/c3np70124g

Barton, D.L., Atherton, P.J., Bauer, B.A., Moore, D.F., Mattar, B.I., LaVasseur, B.I., Rowland, K.M., Zon, R.T., LeLindqwister, N.A., Nagargoje, G.G., Morgenthaler, T.I., Sloan, J.A., Loprinzi, C.L., 2011. The Use of Valeriana officinalis (Valerian) in Improving Sleep in Patients Who Are Undergoing Treatment for Cancer: A Phase III Randomized, Placebo-Controlled, Double-Blind Study (NCCTG Trial, N01C5). J. Support. Oncol. 9, 24-31. doi:10.1016/j.suponc.2010.12.008

Bell, S.A., Niehaus, T.D., Nybo, S.E., Chappell, J., n.d. Structure-Function Mapping of Key Determinants for Hydrocarbon Biosynthesis by Squalene and Squalene Synthase-like Enzymes from the Green Alga Botryococcus braunii Race B. Biochemistry 0, null. doi:10.1021/bi501264s

Benke, D., Barberis, A., Kopp, S., Altmann, K.H., Schubiger, M., Vogt, K.E., Rudolph, U., Muhler, H., 2009. GABAA receptors as in vivo substrate for the anxiolytic action of valerenic acid, a major constituent of valerian root extracts. Neuropharmacology 56, 174-181. doi:10.1016/j.neuropharm.2008.06.013

Bent, S., Padula, A., Moore, D., Patterson, M., Mehling, W., 2006. Valerian for Sleep: A Systematic Review and Meta-Analysis. Am. J. Med. doi:10.1016/j.amjmed.2006.02.026

Bos, R., Woerdenbag, H.J., Hendriks, H., Zwaving, J.H., De Smet, P.A.G.M., Tittel, G., Wikstrom, H. V., Scheffer, J.J.C., 1996. Analytical aspects of phytotherapeutic valerian preparations. Phytochem. Anal. 7, 143-151. doi:10.1002/(SICl)1099-1565(199605)7:3<143::AID-PCA284>3.0.CO;2-1

Bos, R., Woerdenbag, H.J., van Putten, F.M., Hendriks, H., Scheffer, J.J., 1998. Seasonal variation of the essential oil, valerenic acid and derivatives, and velopotriates in Valeriana officinalis roots and rhizomes, and the selection of plants suitable for phytomedicines. Planta Med. 64, 143-147. doi:10.1055/s-2006-957392

Del Valle-Mojica, L., Ortíz, J., 2012. Anxiolytic Properties of Valeriana officinalis in the Zebrafish: A Possible Role for Metabotropic Glutamate Receptors. Planta Med. 78, 1719-1724. doi:10.1055/s$0032-1315240$

Estévez, J.M., Cantero, A., Reindl, A., Reichler, S., León, P., 2001. 1-Deoxy-D-xylulose-5-phosphate synthase, a limiting enzyme for plastidic isoprenoid biosynthesis in plants. J. Biol. Chem. 276, 22901-22909. doi:10.1074/jbc.M100854200

Khan, N., Nybo, S.E., Chappell, J., Curtis, W.R., 2015. Triterpene Hydrocarbon Production Engineered Into a Metabolically Versatile Host - Rhodobacter capsulatus. Biotechnol. Bioeng. n/a-n/a. doi:10.1002/bit.25573

Lee, S.Y., 1996. High cell-density culture of Escherichia coli. Trends Biotechnol. 14, 98-105. doi:10.1016/0167-7799(96)80930-9

Luger, D., Poli, G., Wieder, M., Stadler, M., Ke, S., Ernst, M., Hohaus, A., Linder, T., Seidel, T., Langer, T., Khom, S., Hering, S., 2015. Identification of the putative binding pocket of valerenic acid on GABAA receptors using docking studies and site-directed mutagenesis. Br. J. Pharmacol. 172, 5403-5413. doi:10.1111/bph.13329

Lutz, R., Bujard, H., 1997. Independent and tight regulation of transcriptional units in escherichia coli via the LacR/O, the TetR/O and AraC/I1-I2 regulatory elements. Nucleic Acids Res. 25, 1203-1210. doi:10.1093/nar/25.6.1203 
Martin, V.J.J., Pitera, D.J., Withers, S.T., Newman, J.D., Keasling, J.D., 2003. Engineering a mevalonate pathway in Escherichia coli for production of terpenoids. Nat. Biotechnol. 21, 796-802. doi:10.1038/nbt833

Martin, V.J.J., Yoshikuni, Y., Keasling, J.D., 2001. The in vivo synthesis of plant sesquiterpenes by Escherichia coli. Biotechnol. Bioeng. 75, 497-503. doi:10.1002/bit.10037

Morrone, D., Lowry, L., Determan, M.K., Hershey, D.M., Xu, M., Peters, R.J., 2010. Increasing diterpene yield with a modular metabolic engineering system in E. coli: Comparison of MEV and MEP isoprenoid precursor pathway engineering. Appl. Microbiol. Biotechnol. 85, 1893-1906. doi:10.1007/s00253-009-2219-x

Niehaus, T.D., Okada, S., Devarenne, T.P., Watt, D.S., Sviripa, V., Chappell, J., 2011. Identification of unique mechanisms for triterpene biosynthesis in Botryococcus braunii. Proc. Natl. Acad. Sci. U. S. A. $108,12260-12265$. doi:10.1073/pnas. 1106222108

Peralta-Yahya, P.P., Ouellet, M., Chan, R., Mukhopadhyay, A., Keasling, J.D., Lee, T.S., 2011. Identification and microbial production of a terpene-based advanced biofuel. Nat. Commun. doi:10.1038/ncomms 1494

Picaud, S., Olofsson, L., Brodelius, M., Brodelius, P.E., 2005. Expression, purification, and characterization of recombinant amorpha-4,11-diene synthase from Artemisia annua L. Arch. Biochem. Biophys. 436, 215-226. doi:10.1016/j.abb.2005.02.012

Redding-Johanson, A.M., Batth, T.S., Chan, R., Krupa, R., Szmidt, H.L., Adams, P.D., Keasling, J.D., Soon Lee, T., Mukhopadhyay, A., Petzold, C.J., 2011. Targeted proteomics for metabolic pathway optimization: Application to terpene production. Metab. Eng. 13, 194-203. doi:10.1016/j.ymben.2010.12.005

Ricigliano, V., Kumar, S., Kinison, S., Brooks, C., Nybo, S.E., Chappell, J., Howarth, D.G., 2016. Regulation of sesquiterpenoid metabolism in recombinant and elicited Valeriana officinalis hairy roots. Phytochemistry 125, 43-53. doi:10.1016/j.phytochem.2016.02.011

Rohmer, M., 1999. The discovery of a mevalonate-independent pathway for isoprenoid biosynthesis in bacteria\{,\} algae and higher plants[dagger]. Nat. Prod. Rep. 16, 565-574. doi:10.1039/A709175C

Sambrook, J., W Russell, D., 2001. Molecular Cloning: A Laboratory Manual. Cold Spring Harb. Lab. Press. Cold Spring Harb. NY 999.

Sowden, R.J., Yasmin, S., Rees, N.H., Bell, S.G., Wong, L.-L., 2005. Biotransformation of the sesquiterpene (+)-valencene by cytochrome P450cam and P450BM-3. Org. Biomol. Chem. 3, 5764. doi:10.1039/b413068e

Takemoto, H., Omameuda, Y., Ito, M., Fukuda, T., Kaneko, S., Akaike, A., Kobayashi, Y., 2014. Inhalation Administration of Valerena-4,7(11)-diene from Nardostachys chinensis Roots Ameliorates Restraint Stress-Induced Changes in Murine Behavior and Stress-Related Factors. Biol. Pharm. Bull. 37, 1050-1055. doi:10.1248/bpb.b14-00136

Tsuruta, H., Paddon, C.J., Eng, D., Lenihan, J.R., Horning, T., Anthony, L.C., Regentin, R., Keasling, J.D., Renninger, N.S., Newman, J.D., 2009. High-level production of amorpha-4, 11-diene, a precursor of the antimalarial agent artemisinin, in Escherichia coli. PLoS One 4. doi:10.1371/journal.pone.0004489

Wang, C., Yoon, S.-H., Jang, H.-J., Chung, Y.-R., Kim, J.-Y., Choi, E.-S., Kim, S.-W., 2011. Metabolic engineering of Escherichia coli for a-farnesene production. Metab. Eng. 13, 648-655. doi:10.1016/j.ymben.2011.08.001

Yeo, Y.-S., Nybo, S.E., Chittiboyina, A.G., Weerasooriya, A.D., Wang, Y.-H., Góngora-Castillo, E., Vaillancourt, B., Buell, C.R., DellaPenna, D., Celiz, M.D., Jones, a D., Wurtele, E.S., Ransom, N., Dudareva, N., Shaaban, K. a, Tibrewal, N., Chandra, S., Smillie, T., Khan, I. a, Coates, R.M., Watt, 
D.S., Chappell, J., 2013. Functional identification of valerena-1,10-diene synthase, a terpene synthase catalyzing a unique chemical cascade in the biosynthesis of biologically active sesquiterpenes in Valeriana officinalis. J. Biol. Chem. 288, 3163-73. doi:10.1074/jbc.M112.415836

Zhang, F., Rodriguez, S., Keasling, J.D., 2011. Metabolic engineering of microbial pathways for advanced biofuels production. Curr. Opin. Biotechnol. 22, 775-783. doi:10.1016/j.copbio.2011.04.024

Zhang, H., Liu, Q., Cao, Y., Feng, X., Zheng, Y., Zou, H., Liu, H., Yang, J., Xian, M., 2014. Microbial production of sabinene--a new terpene-based precursor of advanced biofuel. Microb. Cell Fact. 13, 20. doi:10.1186/1475-2859-13-20 
bioRxiv preprint doi: https://doi.org/10.1101/148338; this version posted June 9, 2017. The copyright holder for this preprint (which was not certified by peer review) is the author/funder. All rights reserved. No reuse allowed without permission. 
Methyl erythritol phosphate (MEP)

A 


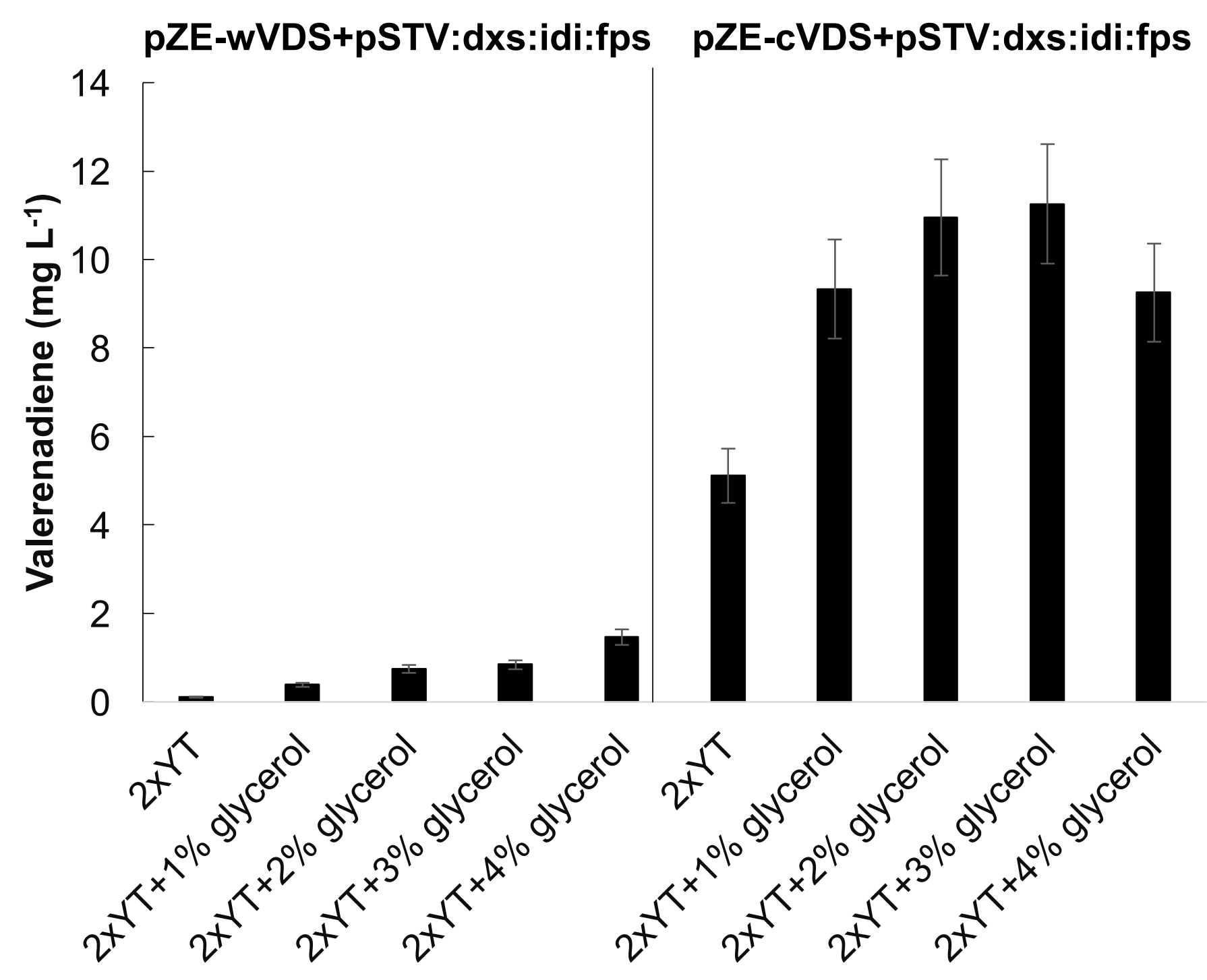



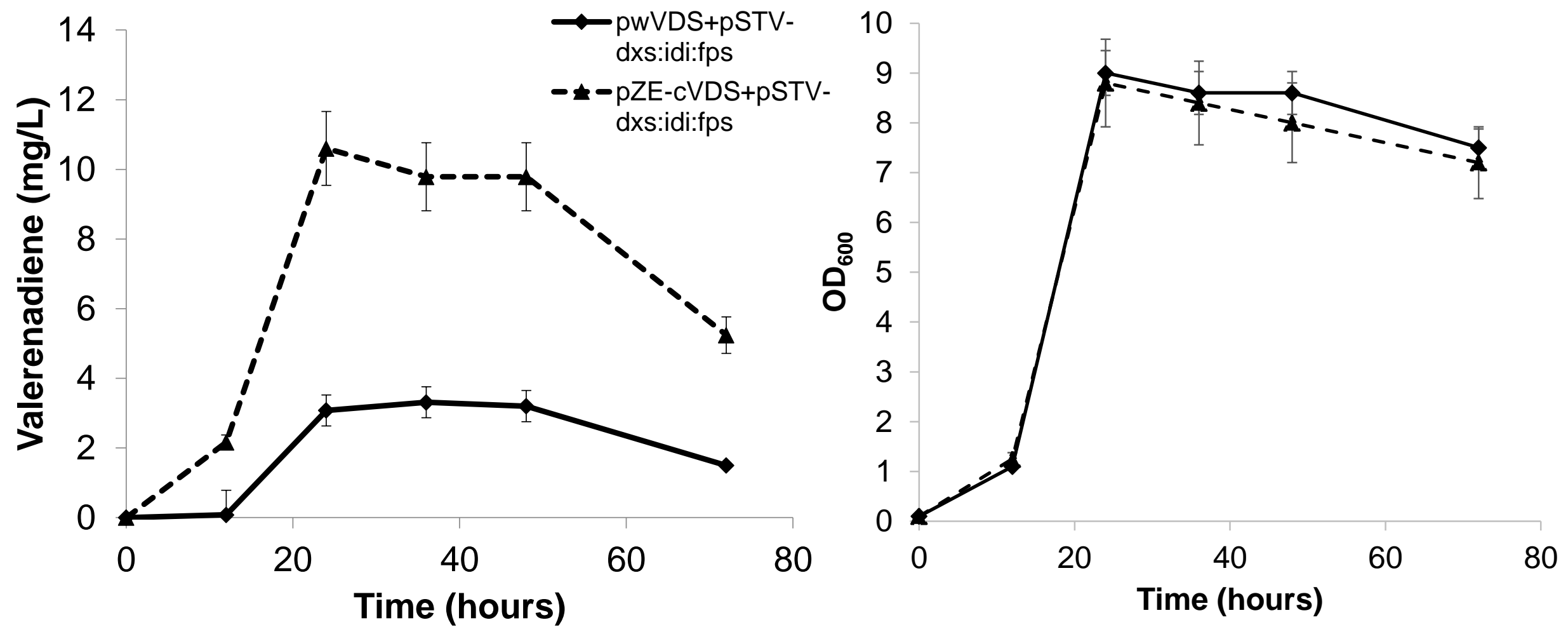

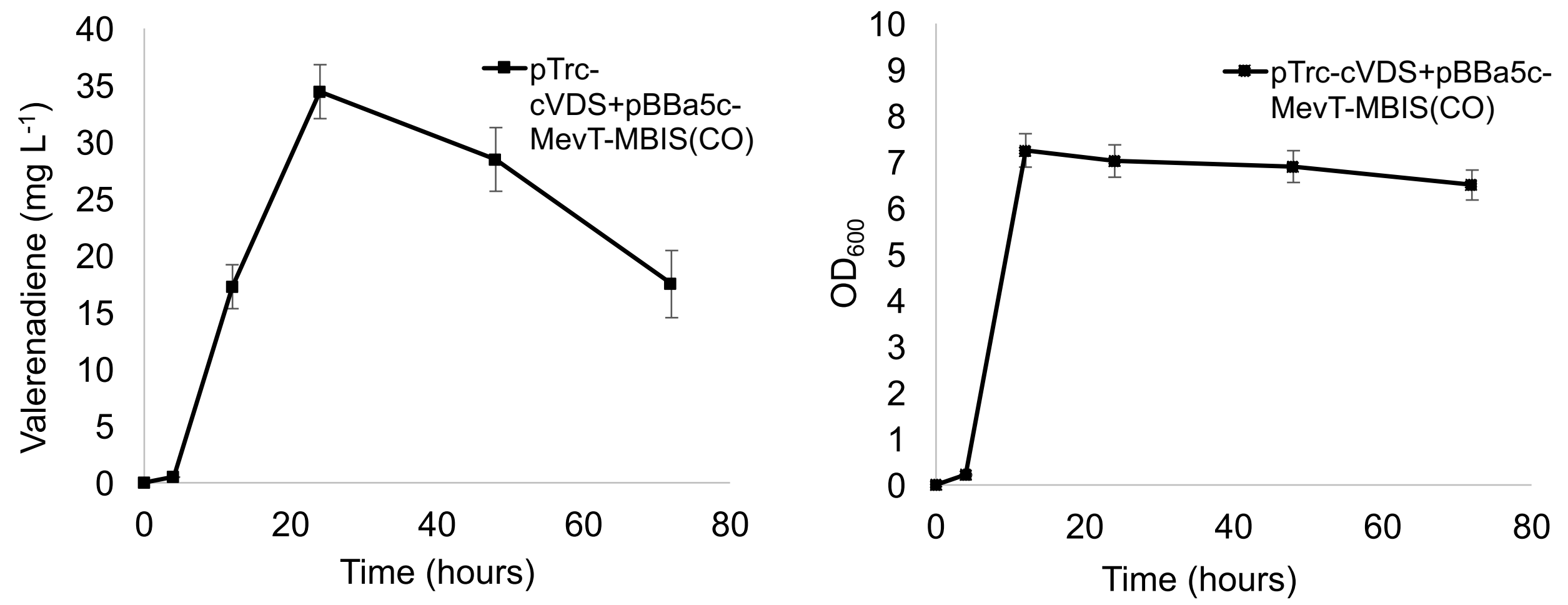

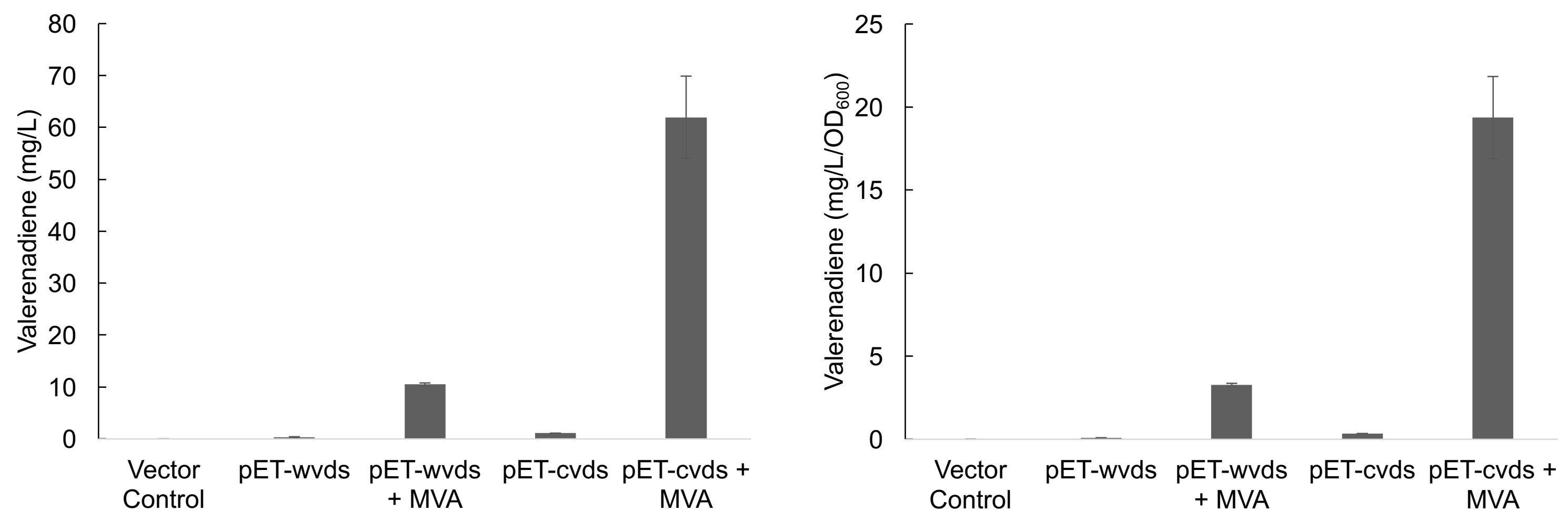